\title{
Research on ship-borne UXB antenna servo system based on LQG controller
}

\author{
Ji Hui $^{1, a^{*}}$, Zhang Xudong ${ }^{2, b}$, and Ji Haiyu ${ }^{3, c}$ \\ ${ }^{1}$ China Satellite Maritime Tracking and Controlling Department, China \\ ${ }^{2}$ China Satellite Maritime Tracking and Controlling Department, China \\ ${ }^{3}$ China Satellite Maritime Tracking and Controlling Department, China \\ a834445867@qq.com, b834324432@qq.com, c53242346@qq.com
}

${ }^{*}$ Corresponding author

Keywords: Servo system; Optimum control; Kalman filter; Gain matrix; MATLAB simulation

\begin{abstract}
Linear Quadratic Gaussian optimal control which is namely LQG control algorithm, is superior to PI control algorithms in control performance, such as adjusting time and overshoot. In closed-loop system, LQG has good anti-interference ability and stability. In order to reduce the pointing error and tracking error, to ensure the direction accuracy and tracking accuracy of the ship borne UXB antenna, the LQG controller is designed and the mathematical model is set up. Finally, using MATLAB software to build a model and the simulation experiment is carried out by based on the designed LQG controller.
\end{abstract}

\section{Introduction}

With the development of deep space exploration, the antenna beam is getting narrower and narrower. Compared with the USB and UCB antenna, the UXB antenna puts forward higher requirements for the pointing precision and tracking precision of the servo system. At present, the antenna servo control system of domestic land station adopts PID control algorithm. The traditional PID controller has many advantages, such as simple structure and design without considering the dynamic performance of the antenna. So it is widely used in the field of control. However the shipborne UXB antenna is affected by the ship-sway and the gust of the outside, the control effect of the traditional PID controller has been difficult to meet the requirement of the direction precision of the UXB antenna.

Linear Quadratic Gauss control algorithm is the optimal control strategy established under the linear two-order Gauss optimal control state space model. The LQG controller consists of the optimal feedback gain controller and the Kalman filter for the system which has a good performance in industry. Compared with the PI controller, the LQG controller has better antiinterference ability and can significantly improve the tracking precision of the antenna under the ship-sway interference.

\section{Design of THE LQG optimal controller}

LQG control is stochastic optimal control under the effect of white noise disturbance of Gauss distribution. According to the principle of separation, this algorithm can be decomposed into 2 aspects, namely linear quadratic optimal control and Kalman filter.

\subsection{Design of linear quadratic optimal control (LQR).}

It is assumed that the state equation of a discrete linear constant system is as follows:

$$
\begin{gathered}
x(k+1)=A x(k)+B u(k) \\
y(k)=C x(k)
\end{gathered}
$$


In the form, $\boldsymbol{A}$ is a matrix of $n \times n$ system, $\boldsymbol{B}$ is a $n \times p$ control matrix, $\boldsymbol{C}$ is a $q \times n$ observation matrix, $\boldsymbol{x}(k)$ is a $n$ dimensional state vector, $\boldsymbol{u}(k)$ is a $p$ dimension input vector, $\boldsymbol{y}(k)$ is a $q$ dimension output vector.

It is required to seek the control vector $\boldsymbol{u}^{*}(t)$ to make the quadratic form object function minimum:

$$
\boldsymbol{J}=\frac{1}{2} \sum_{0}^{N}\left[\boldsymbol{x}^{\boldsymbol{T}}(k) \boldsymbol{Q} \boldsymbol{x}(k)+\boldsymbol{u}^{\boldsymbol{T}}(k) \boldsymbol{R} \boldsymbol{u}(k)\right]
$$

In the form, $\boldsymbol{Q}$ is a semi-positive definite symmetric constant matrix; $\boldsymbol{R}$ is a positive definite real symmetric constant matrix.

Using the method of Lagrange multipliers, the optimal control law can be derived:

$$
\boldsymbol{u}^{*}(k)=-\boldsymbol{R}^{-1} \boldsymbol{B}^{\boldsymbol{T}} \boldsymbol{A}^{\boldsymbol{T}}[\boldsymbol{S}(k)-\boldsymbol{Q}] \boldsymbol{x}(k)=-\boldsymbol{K}(k) \boldsymbol{x}(k)
$$

In the form, $\boldsymbol{K}$ is the optimal feedback gain matrix, $\boldsymbol{S}(k)$ must satisfy the Riccati difference equation:

$$
\boldsymbol{S}(k)=\boldsymbol{Q}+\boldsymbol{A}^{\boldsymbol{T}} \boldsymbol{S}(k+1)\left[\boldsymbol{I}+\boldsymbol{B} \boldsymbol{R}^{-\mathbf{1}} \boldsymbol{B}^{\boldsymbol{T}} \boldsymbol{S}(k+1)\right]^{-\mathbf{1}} \boldsymbol{A}
$$

Therefore, the design of the LQR controller is attributed to the problem of solving the Riccati equation, and find the optimal feedback gain matrix $\boldsymbol{K}$.

Based on the accumulation of equipment data, the input and output relationship is as follows:

$$
\boldsymbol{x}(k+1)=\left[\begin{array}{ccccc}
1.708 & 0 & 1 & 0 \\
-0.7349 & 0 & 1 & 0 \\
-0.0375 & 0 & 0 & 1 \\
0.04807 & 0 & 0 & 0
\end{array}\right] \boldsymbol{x}(k)+\left[\begin{array}{c}
0.8604 \\
-1.718 \\
1.119 \\
-0.2484
\end{array}\right] \boldsymbol{u}(k)+\left[\begin{array}{c}
0.117 \\
-0.8362 \\
1.3505 \\
-0.563
\end{array}\right] \boldsymbol{w}(k)
$$

Select $\boldsymbol{Q}=\operatorname{diag}[10,1,1,1], \boldsymbol{R}=4$. Using the $\operatorname{dlqr}(\boldsymbol{A}, \boldsymbol{B}, \boldsymbol{Q}, \boldsymbol{R})$ functions of MATLAB, calculate the gain matrix $\boldsymbol{K}_{\boldsymbol{u}}=\left[\begin{array}{llll}0.7786 & 0.4726 & 0.03914 & 0.1069\end{array}\right]$.

\subsection{The design of Kalman filter}

For the stochastic control system, the system is disturbed by the process disturbance and the output noise, the observed data is inaccurate due to the influence of random disturbance and output measurement noise. So the state of the system must be reconstructed. Obtaining real and useful data from signals or data contaminated by random disturbance and noise will be accomplished by the Kalman filter.

It is assumed that the discrete state equation of the control object model is as follows:

$$
\begin{gathered}
\boldsymbol{x}(k+1)=\boldsymbol{A} \boldsymbol{x}(k)+\boldsymbol{B} \boldsymbol{u}(k)+\boldsymbol{G} \boldsymbol{w}(k) \\
\boldsymbol{y}(k)=\boldsymbol{C} \boldsymbol{x}(k)
\end{gathered}
$$

In the form, $\boldsymbol{w}(t) 、 \boldsymbol{v}(t)$ are white noise signals, $\boldsymbol{w}(t)$ is system interfering noise, $\boldsymbol{v}(t)$ is system measurement noise, $\boldsymbol{G}$ is a noise gain array.

$$
\begin{gathered}
\boldsymbol{E}\{\boldsymbol{w}(k)\}=\boldsymbol{E}\{\boldsymbol{v}(k)\}=\mathbf{0} \\
\boldsymbol{E}\left\{\boldsymbol{w}(k) \boldsymbol{w}(k)^{\boldsymbol{T}}\right\}=\boldsymbol{Q} \\
\boldsymbol{E}\left\{\boldsymbol{v}(k) \boldsymbol{v}(k)^{\boldsymbol{T}}\right\}=\boldsymbol{R}
\end{gathered}
$$

The state of the estimate is expressed in $\boldsymbol{x}(k \mid k)$, the covariance $\boldsymbol{p}(k \mid k)$ of the estimated state is defined as:

$$
\boldsymbol{p}(k \mid k)=\boldsymbol{E}\left\{[\boldsymbol{x}(k)-\boldsymbol{x}(k \mid k)][\boldsymbol{x}(k)-\boldsymbol{x}(k \mid k)]^{\boldsymbol{T}}\right\}
$$

The estimated value of the state variable $\boldsymbol{x}(k)$ is obtained when the covariance is minimization. The algorithm for the cyclic estimation of the state variable $\boldsymbol{x}(k)$ is as follows:

$$
\boldsymbol{x}(k \mid k-1)=\boldsymbol{A} \boldsymbol{x}(k-1 \mid k-1)+\boldsymbol{B} \boldsymbol{x}(k)
$$

Calculating the error variance of the predicted state $\boldsymbol{p}(k \mid k-1)$ :

$$
p(k \mid k-1)=A p(k \mid k-1) A^{T}+G Q G_{1}^{T}
$$

The calculation method of filter gain matrix $\boldsymbol{K}_{f}(k)$ :

$$
\boldsymbol{K}_{f}(k)=\boldsymbol{p}(k \mid k-1) \boldsymbol{C}^{\boldsymbol{T}}\left[\boldsymbol{C} \boldsymbol{p}(k \mid k-1) \boldsymbol{C}^{\boldsymbol{T}}+\boldsymbol{R}\right]^{-\mathbf{1}}
$$

The variance of calculation error of filter estimation $\boldsymbol{p}(k \mid k)$ :

$$
\boldsymbol{p}(k \mid k)=\left[\boldsymbol{I}-\boldsymbol{K}_{f}(k) \boldsymbol{C}\right] \boldsymbol{p}(k \mid k-1)
$$


There is no disturbance and noise in the system that composed of the States predicted by the Kalman optimal estimator.

Select $\boldsymbol{Q}=1, \boldsymbol{R}=1$. By using the discrete Kalman recursive formula, the convergent filter feedback gain can be obtained:

$$
\boldsymbol{K}_{f}=\left[\begin{array}{llll}
-1.591 & -0.1013 & 1.388 & -0.6111
\end{array}\right]^{T}
$$

\section{Simulation experiment}

By using MATLAB to build a model for the loop of a ship-borne UXB servo system, design the PID controller and the LQG controller respectively. According to the design of the LQG controller, the position loop simulation model based on the LQG controller can be completed, as shown in Figure 1.

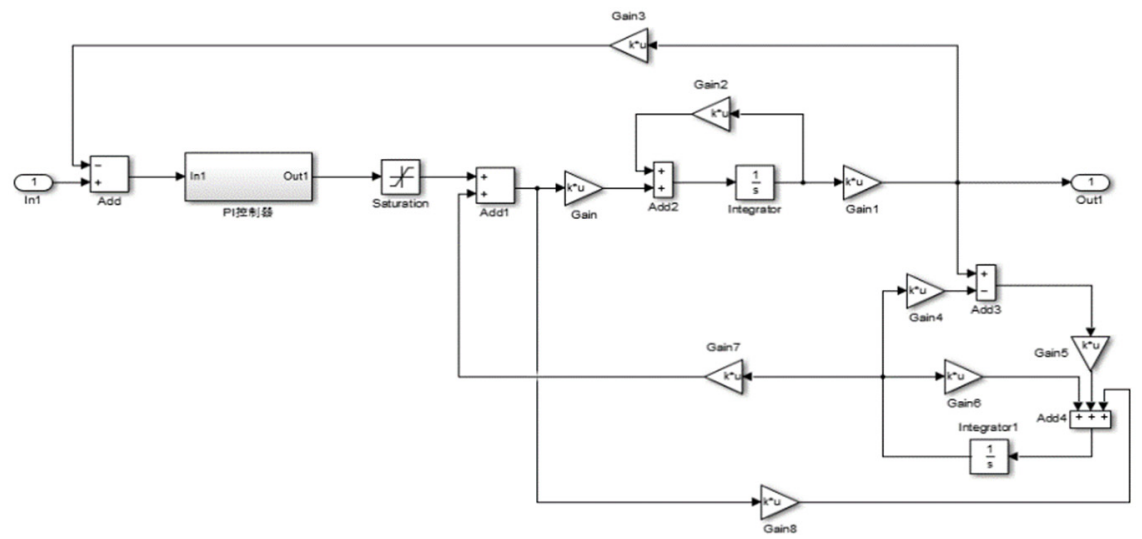

Fig. 1. Position loop model of LQG controller

According to the existing equipment, the position loop based on PID controller is simulated, as shown in Figure 2.

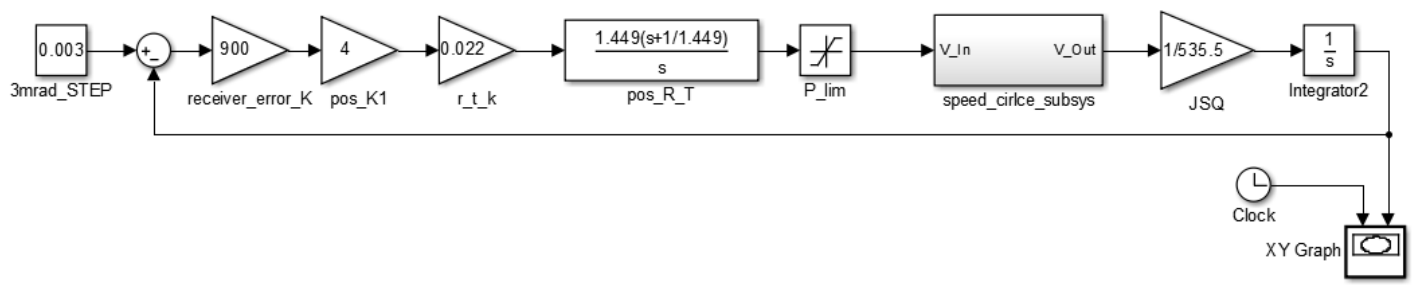

Fig. 2. Position loop model of PID controller.

The step test is carried out on the position loop model of PID controller and LQG controller separately.

Table 1. Performance comparison

\begin{tabular}{cccc}
\hline Controller & Adjusting time & Overshoot & Bandwidth \\
\hline LQG & 0.7 & 0 & 4 \\
PID & 1.3 & $12 \%$ & 1.77 \\
\hline
\end{tabular}

After proper overshoot, the step response of the LQG controller eliminates the error according to the optimal curve. It fully shows the superior performance of the optimal control.

\section{The conclusion}

By analyzing the working principle of LQG controller, the linear quadratic optimal control and Kalman filter are designed respectively. According to the parameters of the ship's UXB antenna servo system, adjust LQR controller and Kalman filter. By using MATLAB simulation, the position loop of servo system based on PID controller and LQG controller is set up respectively. Through 
testing, it is proved that the dynamic performance of the LQG controller is far superior to the PID controller.

\section{References}

[1] LI Lian-sheng. Modern radar servo system ,M. Beijing: national defence industry press, 2003.

[2] Square-Root Cubature-Quadrature K alman Filter[J] . Asian J Control . 2014 (2)

[3] Zhang Zhiyong. Proficient in MATLAB (M). Beihang University press, 2000.

[4] Han Yong Qiang, Chen Jia Bin. Unscented Kalman Filter for DR/GPS Integrated Navigation System[J] . Advanced Materials Research . 2010 (121)

[5] Zdzislaw Duda. State estimation in a decentralized discrete time LQG control for a multisensor system [J]. Archives of Control Sciences. 2017(1).

[6] Jiankui Chen, Maozheng Chen. Application of LQG Controller in Antenna Servo Control System. Third IEEE International Conference on Information Science and Technology (ICIST2013).

[7] Arasaratnam, Ienkaran. Cubature Kalman filtering for continuous-discrete systems: Theory and simulations. IEEE Transactions on Signal Processing . 2010

[8] Weidong Wang; Yijuan Jing; Li Yang; Boyuan Ma; Zhengguo Fu. Weight Optimization for LQG Controller Based on the Artificial Bee Colony Algorithm. Proceedings of 2012 Conference on Modelling, Identification and Control(MIC 2012 V3).

[9] Kaiyu Hu, Aili Yusup, Wenwen Chang. Simulation and Analysis of LQG Controller in Antenna Control System. Proceedings of 2013 IEEE 4th International Conference on Electronics Information and Emergency Communication.2013.

[10]Lingjiang Chai,Tao Sun,Jerzy Warminski. The Design of LQG Controller for Active Suspension Based on Analytic Hierarchy Process[J] . Mathematical Problems in Engineering . 2010.

[11]Chen Shian,Zhou Hang,Liu Hongguang,Yao Ming.Optimal design of active suspension based on LQG control without road input signal. 4th International Conference on Intelligent Computation Technology and Automation,ICICTA 2011 . March 28 2011-March29 2011.

[12]Roger Moliner,Rafael Tanda. Herramienta para la sintonía robusta de controladores PI/PID de dos grados de libertad[J]. Revista Iberoamericana de Automática e Informática Industrial. 2016(1)

[13]Arrieta,R. Vilanova. Simple PID tuning rules with guaranteed M s robustness achievement[J]. IFAC Proceedings Volumes. 2011(1).

[14]Masahiko Mori,Kazuo Yamazaki,Makoto Fujishima,Jiancheng Liu,Norio Furukawa. A Study on Development of an Open Servo System for Intelligent Control of a CNC Machine Tool[J] . CIRP Annals-Manufacturing Technology . 2001 (1).

[15] W.J.Liao,S.H.Chang,J.T.Yeh,et al. Compact dual-band WLAN diversity antennas on USB dongle platform. IEEE Transactions on Antennas and Propagation . 2014. 